\title{
Für eine Re-Sozialisierung des Helfens
}

\author{
Klaus Dörner löst eine Debatte um Professionalität, \\ Sozialwirtschaft und Bürgergesellschaft aus
}

Albert Mühlum

Der Markt ist prinzipiell das falsche Koordinatensystem für das Gesundheits- und Sozialwesen, stellt der Psychiater und $\mathrm{Hi}$ storiker Prof. Dr. Klaus Dörner in seinem Buch zur » Gesundheitsfalle " fest. Kritisch geht er dabei auch mit den »Profi-Helfern« ins Gericht.

»Starker Tobak « - so der erste Eindruck. Wäre da nicht der prominente Autor und seine unbestreitbare Expertise für Fragen der Gesundheit und Krankheit, hätte der reißerische Titel vermutlich abgeschreckt. Ein zweiter Blick weckt das Interesse: Markt-Doping, Bürger-Helfer, Gesundheitsfalle und ein Befreiungsprogramm wie mag das zusammenpassen? Im Vorwort erste Klärungen:

- Professionelles Helfen wird dem mitmenschlich-nachbarschaftlichen Helfen gegenüber oder richtiger: zur Seite gestellt.

- Doping bedeute, dass sich die ProfiHelfer mit dem Markt solcher Mittel bedienen, die eigentlich verboten sind und Menschen schädigen können, nur um die eigene Leistung zu steigern.

- Gesundheitsfalle meint die quantitative Ausdehnung des Sozial- und Gesundheitssystems mit schädlichen, statt positiven Folgen für die Gesundheit.

Weiter: »Perversionskrise des Profi-Hilfesystems «, » marktbedingte Korruptionskrise « (S. 10); Geld fließe »nicht mehr zu den Hilfebedürftigsten, sondern eher zu

Prof. Dr. Albert Mühlum war bis zu seiner Pensionierung Hochschullehrer für Sozialpolitik und

Sozialarbeitswissenschaft an der Fachhochschule Heidelberg und Lehrbeauftragter der Universität Heidelberg. Er war lange Jahre Vorstandsmitglied der Deutschen Gesellschaft für Soziale Arbeit e. V. E-Mail a.muehlum@t-online.de den profitableren Gesünderen « und »alle Welt verkauft Gesundheit als höchstes Gut, um unter dieser Tarnung umso erfolgreicher alles Gesunde ... in Krankes und damit Behandlungsbedürftiges $\mathrm{zu}$ verwandeln «(S. 8) - was das Hilfesystem stärker als alle anderen Branchen boomen lasse, die Profi-Helfer aber korrumpiere.

Der Autor räumt ein, dass das Buch unausgewogen ist, weil er sich darauf beschränkt, "nicht die ohnehin akzeptierten Vorteile der Profi-Hilfe noch mal zu akzeptieren, sondern die vernachlässigten Nachteile und Gefahren stark zu machen - vorsichtshalber manchmal zu stark.«(S. 9) Dazu wurden frühere Publikationen (»Die Gesundheitsfalle « 2003, »Das Gesundheitsdilemma « 2004) überarbeitet, zugespitzt und auf das gesamte Profi-Hilfesystem übertragen.

Die Gliederung verstärkt den Eindruck eines leidenschaftlichen Plädoyers für eine neue Kultur des Helfens. Bewusst werden auch die Emotionen des Lesers angesprochen. So beginnen fast alle Kapitelüberschriften mit »Wir Profi-Helfer«. Sie reichen von einer kritisch-polemischen Bestandsaufnahme (»Wir Profi-Helfer werden durch das Markt-Doping zu Gesundheitsvernichtern dressiert « (1), ".. sind keine Helfer mehr ...«(2), »... programmieren die Bürger nach den Leitbildern des homo oeconomicus ... «(3) über einige Zwischenschritte (»Wir Profi-Helfer wollen die Bürgerhelfer ersetzen können sie aber nur ergänzen « (4), »... reiben uns die Augen: alle Menschen sind auch helfensbedürftig « (5), »... haben die Unheilbaren zu lieben ...«(6), »Mit Fehlerfreundlichkeit ist das Markt-Doping von uns Profi-Helfern wenigstens zu unterlaufen ...« (7) bis zu strategischen Aufforderungen (»Hausärzte als Mut machendes Modell ...«(8), "Wir ProfiHelfer müssen wollen, dass möglichst viele Bürger auch Bürgerhelfer und damit unsere Konkurrenten werden « (9), »... vereinigen uns mit der neuen Bürgerhelferbewegung im dritten Sozialraum zum
Bürger-Profi-Mix « (10), »Auch in der Wissenschaft holen uns Profis vielleicht nur die Bürger aus der markt-gedopten Gesundheitsfalle« (11), »Nur unser Bürger-Profi-Bündnis weist allen Helfenden den Letzte-Chancen-Weg zur > Würde der Gesunden« (12). Ohne die Kapitel im einzelnen auch nur annähernd würdigen zu können, seien einige Aspekte hervorgehoben:

Zunächst das Gesundheitsverständnis des Autors: Er folgt nicht der idealistischen WHO-Definition, sondern begreift mit Gadamer Gesundheit als das »selbstvergessene Weggegebensein«, das gerade nicht machbar sei, sondern Geschenk. Gesundheit erweise sich an den Grunddimensionen, in denen sich Menschen verausgaben können: körperliche und geistige Leistung, genuss- und lustvolle Kreativität sowie Liebe und Sorge für Andere, also Leistungsfähigkeit, Lebenslust und Verantwortungsbereitschaft.

Zur Gesundheitsfalle gehörten Denkfehler der Profi-Helfer wie beispielsweise der Irrglaube, Krankheiten besiegen und Gesundheit oder gar eine leidensfreie Gesundheitsgesellschaft herstellen zu können. Im Übrigen werde die verbreitete Fixierung auf Gesundheit diese dauerhaft beeinträchtigen. Das gilt auch gesamtgesellschaftlich: "Ein Krankheitsbewältigungssystem, das als Gesundheitssystem sich ... grenzenlos steigern will, wird zur Gesundheitsvernichtungsmaschine« (S. 13).

Ein wiederkehrender Topos ist die Professionalisierung und Institutionalisierung des Helfens, die mit der Moderne einsetzt und strukturell notwendig ja segensreich war, aber längst über die Maßen ausgedehnt wurde und u. a. zur »moralischen Verarmung « der Bürger führte: Mit der Sorge für andere und deren Ent-Sorgung in Heime entfielen auch soziale Anerkennung und Bindung, was den »helfensbedürftigen « Menschen selbst und die Gesellschaft (be-) schädige (»soziale Atrophie «). Den Profi-Helfern wiederum falle 
es schwer, sich der »Enteignung der Gesundheit « (= aus Gesunden therapiebedürftige Kranke zu machen) zu widersetzen, wie die verbreitete »Überdiagnostik « und »Übertherapie« belege. So hätten die die Belastung und Entlastung der Menschen aus der Balance geraten sei, obwohl ein gelingendes Leben auch der Last bedürfe (S. 113 ff.), beispielsweise der Sorge für andere oder Schmerz und Leid als

\section{»Dörner hält ein leidenschaftliches Plädoyer für eine neue Kultur des Helfens"}

Profi-Helfer heute etwa zehnmal mehr Bürger zu psychisch Kranken gemacht als vor 40 Jahren und unsere psychosomatischen Reha- und Kur-Kliniken hätten etwa so viele Betten wie die ganze übrige Welt. Wie weit das »markt-gedopt « verursacht ist, wie Dörner meint, oder durch zivilisatorische Belastungen, geschärfte Wahrnehmung und politische Reaktionsformen, ist eine der kritischen Fragen, die sich aufdrängen.

Historisch sieht er einen folgenreichen Übergang von der Sozialgesellschaft (selbstverwaltetes Helfen) zum Sozialstaat (Daseinsfürsorge, Profi-Helfer) und - nach dessen Rückzug - zur Sozialwirtschaft, die er durch marktliche Elemente dominiert sieht. Diese »Marktfalle« bevorzuge die Stärkeren (günstige Prognose) und sei kostentreibend. Steuerungsversuche wie DMP (disease management program) und DRGs (diagnostic related groups) hält er für potenziell patientenschädigend und Qualitätsmanagement für ein grandioses Selbsttäuschungsmanöver. Aber Staatsversagen und Marktversagen hätten inzwischen den Blick für die Grenzen der Ökonomisierung und für die Bedeutung der Bürger-Helfer geschärft.

Der zentrale Vorwurf lautet: Die Übertragung der Marktgesetze auf das Medizin- und Sozialsystem hat ein "MarktDoping-Zwangssystem « geschaffen, dem sich kaum jemand entziehen könne: »Wir Profi-Helfer müssen unsere diagnostischen und therapeutischen Gesundheitsfallen stellen, und die übrigen Bürger müssen darauf hereinfallen und so ihre Gesundheitszeche zahlen« (S. 31f.) wozu das Leitbild des homo oeconomicus mit den Unterformen homo hygienicus und praeventicus erheblich beitrügen, mit dem paradoxen Ergebnis, dass das Leiden umso mehr zunehme, je mehr versucht wird, es zu verringern. Dazu passt, dass
Voraussetzung persönlicher Reifung. Bleibt die entscheidende Frage: Ist der Markt prinzipiell das falsche Koordinatensystem für das Gesundheits- und Sozialwesen, wie Klaus Dörner argumentiert, oder gibt es auch hier Bereiche, in denen Marktprinzipien nützlich oder sogar unverzichtbar sind?

Dörner selbst bekennt sich zur Marktwirtschaft, hält sie jedoch mit Blick auf das Hilfesystem für untauglich. Er verkennt dabei wohl, dass es auch eine Theorie der unvollkommenen Konkurrenz gibt, wonach selbst unter limitierenden Bedingungen positive Effekte möglich sind. Seine ökonomischen Begründungen schießen manchmal über das Ziel hinaus, so beispielsweise seine Begründung, weshalb der Kranke kein Konsument sei: «auf Krankheit könne man i. d. R. nicht verzichten wie auf Konsumgüter« (S. 45). Tatsächlich geht es um Sach- und Dienstleistungen, die angeboten und nachge-

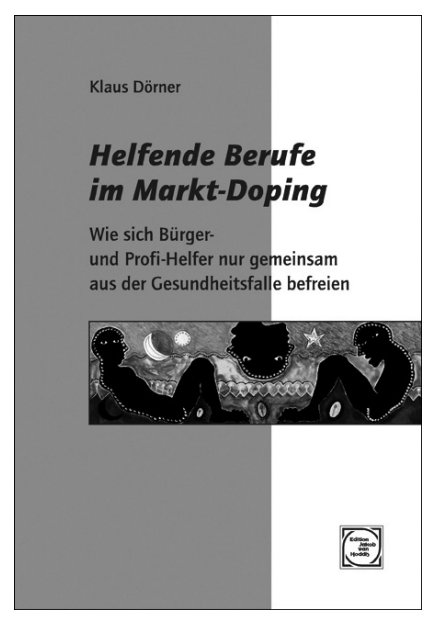

Klaus Dörner: Helfende Berufe im Markt-Doping. Wie sich Bürger- und Profi-Helfer nur gemeinsam aus der Gesundheitsfalle befreien. Paranus-Verlag, Edition Jakob van Hoddis, Neumünster 2008, 172 S., 16,80 Euro. ISBN 978-3926200-98-3. fragt werden, hier mit Blick auf Krankheitsbekämpfung oder Gesundheitsförderung. Eher überzeugen seine Zweifel an der Selbstbestimmung (-sfähigkeit) von Patienten, woraus er eine besondere Profi-Verantwortung ableitet (S. 90 ff.). Eine ganz andere Frage ist bei der unbestreitbaren ökonomischen Dominanz, ob die Gewichte und Rahmenbedingungen stimmen, ob der Marktmechanismus funktioniert und ob der Gleichgewichtspreis » zumutbar « ist, - womit die Sozialpolitik ins Spiel kommt. Sie wäre gut beraten, Profiund Bürgerhelfer zusammen zu bringen, wie Dörner zu Recht fordert: alltagsnah, lokal und ambulant im »dritten Sozialraum« Nachbarschaft (S. 142 f.).

\section{Resümee}

Klaus Dörner legt - sprachmächtig und engagiert wie eh und je - eine polemische Schrift vor: mit oft vergessenen Wahrheiten, gewollt provozierend, stets anregend, manchmal überschießend, aber immer werbend für das genuin Humane. Eindrucksvoll sind die anthropologischphaenomenologischen Betrachtungen, historisch ausgedeutet, mit Belegen für Fehlentwicklungen, Irrwege und Folgerungen für eine »Re-Sozialisierung des Helfens « durch Überwindung einer oft krankheitswertigen Selbstbezogenheit in der " postsäkularen « Gesellschaft.

Kritisch ist anzumerken, dass er ein verengtes Verständnis von Sozialwirtschaft hat und ein kompletter Verzicht auf Marktprinzipien unrealistisch ist, da auch positive Effekte wie Wettbewerb und Leistungsanreize zur Schonung und optimalen Allokation der Ressourcen beseitigt würden. Mancher Vorwurf an den Markt müsste eigentlich der Politik gelten, da es oft staatliche Eingriffe sind, die kontraproduktiv wirken und Kolateralschäden verursachen. Zu kurz kommen Bürokratie, öffentliche Meinung, Macht der Medien und der mündige Konsument. Dennoch ist seinem Plädoyer für die Aufwertung der Sozialgesellschaft und für ein Arbeitsbündnis der Bürger- und ProfiHelfer zuzustimmen, da nur so der künftige Gesamthilfebedarf (S. 169) gedeckt werden kann.

Fazit: Ein wichtiges Buch, das die Debatte in der Sozialen Arbeit um Professionalität, Sozialwirtschaft und Bürgergesellschaft nur bereichern kann. 